\title{
Histological Structure of Anterior Cruciate Ligament - Review
}

\author{
Jahira Banu', Yogesh Sontakke ${ }^{2}$, Dharmaraj Tamgire ${ }^{2}$, Suma H Y ${ }^{3}$ \\ ${ }^{1}$ Junior Resident, JIPMER, ${ }^{2}$ Associate Professor, corresponding Author, JIPMER, ${ }^{3}$ Additional Professor, JIPMER
}

\section{Abstract}

Introduction: Anterior cruciate ligament (ACL) is one of the commonly injured ligaments of the knee joint due to sports activities. Because of the poor healing capacity of the ACL, surgical treatment for ACL injuries was followed for many years. Therefore, understanding the structural knowledge of the ACL will help in reproduce the native ACL. Objectives: To improve the histological knowledge of ACL and to understand the valuation of histology of ACL attachment to the bone. Subjects and Methods: PubMed and Google search was used as a search engine to collect the concerned articles that describing the histology of ACL. The key words were ACL, histology, Ultrastructure. Results: Ultrastructure of ACL observed from proximal to distal attachments showed the more complicated and complex arrangement of collagen bundles with interspersed cells in between. Ultrastructure of ACL also should be borne in mind before preparing ACL grafts. Conclusion: ACL has complex histological structure. It is essential to consider the details of the ACL histological structure in ACL reconstruction surgeries to restore its full functionality. This review may be useful as a reference to investigate the mechanical properties of ACL footprint.

Keywords: Anterior cruciate ligament, Ultrastructure, histology.

Corresponding Author: Dr. Yogesh Ashok Sontakke, Associate Professor, JIPMER, Puducherry,

Received: February 2019

Accepted: February 2019

\section{Introduction}

The ligament is a connective tissue helps in connecting bone to bone. The flexibility and strength of the joints are maintained by the viscoelastic properties of the ligaments. It takes up the tensile load applied to the particular joints. Forces from the ligament are transmitted to the bone via the fibrocartilage zone. Although ligament appear as single structure, with the movement of joint, few fibers of ligaments appear tighten or loosen depending upon the forces applied to them. Injury to the ligaments results in a drastic change in its structural and functional behavior. ${ }^{[1]}$

Cruciate ligaments in the knee joint play a pivotal role in maintaining the stability of the knee joint. They crisscross each other in oblique fashion in the center of the joint. The cruciate arrangement is useful for producing rotatory movements of the knee joint. Anterior cruciate ligament (ACL) controls the anterior movement of tibia over the femur and intercepting the hyperextension of the knee joint. ${ }^{[2]} \mathrm{ACL}$ is attached proximally to the posteromedial aspect of the lateral femoral condyle and distally to the anterior intercondylar region of the tibia.

ACL consists of two bundles, namely, an anteromedial and posterolateral bundle based on their attachment over tibia. Fibers of the anteromedial bundle run from the most proximal part of femoral attachment of the ligament to the anteromedial area of the medial tibial eminence. The posterolateral bundle fibers run distally from the femur to the posterolateral part of the tibial insertion. ${ }^{[3]}$ Ultrastructure of ACL is composite and consists of more collagen bundles arranged in different directions along the length of ACL with cells interspersed in between the bundles. The variable arrangement of the collagen bundles in ACL is useful for its functional activities.

The incidence of ACL injury was on rise because of sports activities. ACL is the most commonly involved structures in the knee joint accounting for about $60 \%$ of knee injuries. ${ }^{[4]}$ ACL injuries prone to functional instability of the knee joint. The tension patterns of intra-articular structures such as capsule, posterior cruciate ligament, as well as extraarticular structures such as medial collateral and lateral collateral ligaments of knee joints, also deranged following ACL injury. Injury to ACL can lead neuromuscular incoordination due to the afferent flow from the knee to the central nervous system. ${ }^{[6]}$ Inspite of replacement of ACL as treatment option, failures do occur, because the replaced graft does not simulate the original structural properties of ACL. A complete anatomic reconstruction involves several prerequisites that have been defined, such as the functional restoration of the ACL to its native dimensions, collagen orientation, and insertion sites. Therefore, understanding the ultrastructural properties of ACL is useful in assessing the treatment options as well as helpful in providing functional stability to the patients. Hence, this review was undertaken to unfold the structural complexity of the ACL and make the reader aware of it.

\section{Subjects and Methods}




\section{Banu et al; Htistalagical Structure of Anteriar Cruciate Ligament}

Search engine: PubMed, Google search

\section{Inclusion and exclusion criteria:}

Articles searched in PubMed and google search as ACL and histology, ACL and immunohistochemistry, ACL and ultrastructure, ACL and Anatomy. Articles in English language were included in this study and articles in other languages were excluded. Articles involving human studies were included and animal study were excluded.

\section{Review}

The present review is described under the following headings: gross features of ACL, light microscopy and ultrastructure, functional role of each components and histopathological changes of ACL. The histopathological alterations in ACL during injuries, that include disruption of collagen bundles and mucoid degeneration has also been discussed.

\section{Light microscopy of Anterior Cruciate Ligament}

The structure of the ACL, like other fibrous connective tissues, comprised a fibrous matrix containing cells, blood vessels and nerves. In contrasts to most connective tissue, the ACL had a more complex histological arrangement. Its primarily consisted of two bundles namely an anteromedial (AM) and a posterolateral bundles (PL) separated by vascularized connective tissue. Each bundle of ACL consisted of collagen fascicles, covered by synovial sheath and each individual fiber was separated by loose connective tissue.

The collagen fascicle ensheathed by loose connective tissue called paratenon and the subunit of collagen fascicle consists of multiple collagen fiber covered by dense connective tissue called epitenon. The endotenon was made up of loose connective tissue covers the collagen fibril, the subunit of collagen fiber. ${ }^{[6-8]}$

\section{Gross structure}

\section{Collagen arrangement in ACL}

The ACL composed of numerous collagen fascicles separated by columns of cells in fibrous capsule. The collagen fascicles range from $250 \mu \mathrm{m}$ to several millimetres and each fascicle organized with 3-20 subfasciculi. Approximately 950 collagen fascicles were seen in ACL. Cross sectional area of each fascicle is $9.4 \times 10-2$ to $48.1 \mathrm{x}$ 10-2 mm2.The sub-fasciculi contain groups of sub-fascicular units that was $100-250 \mu \mathrm{m}$ in diameter. The diameter of collagen fibre was 1-20 $\mu \mathrm{m}$ and that of collagen fibril was $25-250 \mathrm{~nm}$. The fibril density varies among the entire length of ACL. Density of fibril was more in proximal and middle region of ACL. Fibril diameter was known to indicate specific fibril function. ${ }^{[6,9]}$

The arrangement of collagen and its type was varying at different region. Collagen fascicles were oriented at various angles to the long axis of the ligament and a few fascicles run parallel to long axis. Parallel arrangement of collagen fibers with unidirectional course and lamellar arrangement were found in the center of ACL and interlacing fibrils on outer zone of ACL with uniform undulating collagen fibril bundles on the surface with many crossing fibril bundles and triangular distributions of collagen fibers on the attachment region. ${ }^{[9]}$
Most abundant type of collagen observed in ACL was type I collagen. Other types of collagen seen in ACL, includes type III, IV, VI collagen. Arrangement of different types of collagen on ACL was based on the functional adaptation of the ligament. Type I was seen in fascicles and type II in connective tissue coverings. The collagen bundles arranged as planar, parallel and twisted nonlinear networks patterns. The non-linear arrangement of collagen fibers was explained as "Crimp", that indicated the regular sinusoidal pattern of collagen bundle arrangement. ${ }^{[6,7,13,23]}$

Triantafyllidi E et al found out the collagen fibers arranged in a parallel fashion in the center of ACL. They explained the existence of transition area near the attachment zone. The transition zone in histology showed the presence of fibro-cartilaginous and cartilaginous zones. ${ }^{[12]}$ Suzuki et al described the presence of difference in diameter and the profile of collagen fibrils among the bundles of ACL. They divided the ACL as three bundles as anteromedial-medial bundle (AM-MB), anteromedial-lateral bundle (AM-LB) and posterolateral bundle (PLB). The fibers in the posterolateral bundle was different from the AM-MB and AM-LB fibers. Random arrangement of fibers is predominant in PLB fibers and AM-MB fibers were arranged longitudinally. The fibril diameter distribution of AM-MBs showed a bimodal distribution because of the presence of small diameter and large diameter. The fibril diameter of AM-LBs and PLBs showed only one mode of distribution. The fibers of AM-MB thick predominantly and unidirectional in orientation, the PLB is thinner and arranged in random direction whereas AM-LB showed an intermediate structure. ${ }^{[28]}$

Skelley et al described the microstructural properties of ACL as two bundle in different loading conditions. They demonstrated the collagen fiber organization was inhomogeneous and realignment of fibers occur in the distal region of ACL. The AM bundle had stronger collagen fiber alignment in linear fashion, that explains the failure rate within this tissue. They insisted that a bio scaffold or synthetic graft used for treatment should have more material properties and fiber alignment in the AM bundle. ${ }^{[29]}$

Ferretti M et al, studied the bundles of ACL in foetus. They described the gross morphology of the ACL in foetuses was similar to that reported in adults; in contrasts with the histology of Adult ACL, the ACL of foetus was diverse in cellularity and vascularity. ${ }^{[11]}$

\section{Elastic fibers arrangement}

The ACL found to contain numerous elastic components and concentrated more in the interfasicular region. Elastic fibres were arranged in a fine, twisting meshwork either parallel or perpendicular to the long axis of the ligament. ${ }^{[6]}$

Elastic components included oxytalan, elaunin, mature elastic fibers and elastic membranes. Elastic fibers were concentrated on the center of ACL. ${ }^{[6]}$ The Oxytalan fibers were found as electron dense filaments about $11 \mathrm{~nm}$ diameter around the pericellular region and accounts for about $63 \%$ of elastic components. The mature elastic fibers were seen along the periphery and constitute about $25 \%$ of elastic components. The elaunin fibers constitute to $8 \%$ and $4 \%$ by elastic laminae. The elastic laminae were detected by them as a special component in the proximal and distal 
attachment zones of ACL. ${ }^{[9]}$

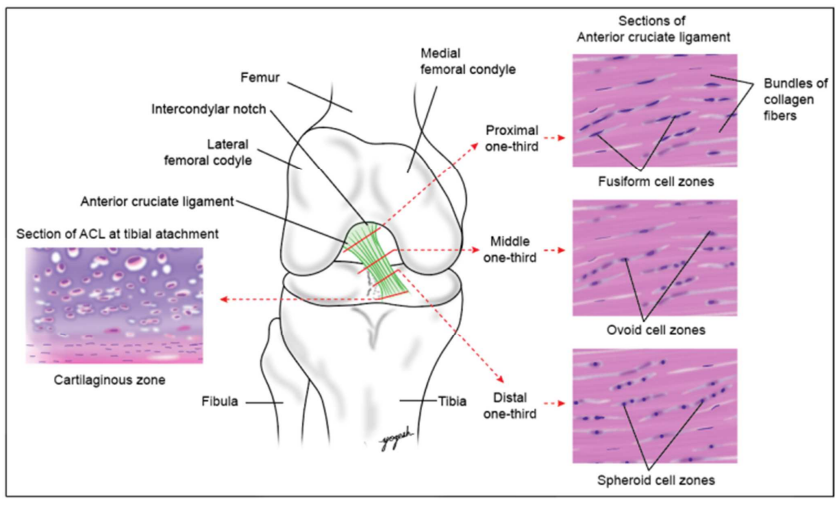

\section{Fibroblast arrangement}

Murray M M et al, noticed the presence of fibroblasts cell among the bundles of collagen and reported the presence of three principal cell types along the length of ACL based on the appearance of nucleus and the presence of lacunar space: fusiform, ovoid, and spheroid. Fusiform, or spindle-shaped cells were related to the crimped collagen fibres and distributed throughout the mid-substance of the anterior cruciate ligament. These cells had also been found in the outer zones of human anterior cruciate ligaments. Ovoid cells had been identified in columns and in lacunae and in central areas of the human anterior cruciate ligament. Spheroid cells were noted within and among the fascicles as well as within the lacunae near the distal area of ACL. ${ }^{[23]}$

Petersen $\mathrm{W}$ et al, described the presence of different cell morphology compared with the other areas of ACL in the anterior part of anteromedial bundle of ACL. The cells in this region resembles typical of tenocyte or chondrocyte cells. They explained that the presence of this cells in this could be the result of impingement of ACL to the anterior rim of intercondylar fossa. ${ }^{[3]}$

\section{Ground substance}

The ACL was found to contain considerable amount of proteoglycans and glycosaminoglycan.

The amount of ground substance was two to four times more when compared with the tendons. ${ }^{[8]}$

\section{Tibial insertion of ACL}

The information on tibial histology will be useful for accurate tibial bone tunnel within the dense ACL attachment during reconstruction surgery and minimize the risk for iatrogenic injuries of adjacent tissue associated with tibial bone tunnel drilling in anatomical ACL reconstruction. The ACL near the tibial insertion area was blended with the lateral meniscus, called the anterior cruciate ligament and lateral meniscus complex (ACL-LM). The attachment of ACL over the tibia was wider than the posterior regions. ${ }^{[14]}$ Furumatsu $\mathrm{T}$ et al used safranin $\mathrm{O}$ stain to differentiate the regions of tibial attachment site. They described the four different zones such as the ligamentous zone, uncalcified and calcified fibrocartilage, and subchondral zone. These zones in sagittal section showed the layered cartilaginous insertion called the direct insertion, that would explain the transition of ligamentous tissue to the bone. The most ventral part of the ACL insertion over the tibia was outlined by a bony anterior ridge. The thickness of the layered direct insertion of ACL was observed to be thinner from medial to lateral. ${ }^{[3]}$

Wang $\mathrm{H} \mathrm{J}$ et al demonstrated the staining pattern of ACL attachment merge with cartilage of the medial tibial plateau in the tibial attachment and characterize the tibial footprint histologically. They stained with hematoxylin and eosin (H\&E) in order to observe the morphology of the ACL insertion and also with fast green and Safranin-O to evaluate for collagen and proteoglycans (PG). They divided the insertional zones into five distinct areas from medial tibial spine to lateral margin of ACL attachment. They numbered the zones from one to five. They observed strong staining pattern to PG and cuboid basketweave arrangement of collagen fibres with densely packed round shaped fibrocartilage cell (FC). Zone 3 showed the staining pattern of longitudinal collagen organization parallel to fiber orientation with fewer FC and lower peak colour of staining compared to zone 1 and Zone 5 shows the staining pattern of scarce interspersed FC and disorganized collagen orientation with staining only at the adjacent area of the tibial footprint. The medial portion of the tibial insertion area showed the increased staining of $\mathrm{PG}$, with more densely organized collagen arrangement and more fibrocartilage cells under high magnification. ${ }^{[19]}$

\section{Femoral insertion of ACL}

The histology of ACL femoral origin showed areas of less dense connective tissue among the transitional zone of ligament and cartilage. Two types of insertion of the fibres of the ACL at their femoral attachment were described by Iwahashi et al a 'direct type' with characteristic zonal architecture, allowing for the gradual dissipation of forces, and an 'indirect type' in that the ligament is inserted into bone by collagen fibres without a transitional zone. ${ }^{[10,16,17,22]}$

\section{Mechanoreceptors in ACL}

Schultz et al described the presence of fusiform mechanoreceptor, axons within the substance of the ligament, and a mechanoreceptor resembling a Golgi tendon organ. ${ }^{[23]}$ A modified gold chloride method staining on ACL used for identifying the mechanoreceptors. Serial sections taken from the ACL was observed under light microscope and the receptors seen on the ACL were photographed. Nerve fibres was found to reaching the ACL via the neurovascular bundle from the connective tissue and terminated in various receptors. Two different mechanoreceptors were identified in addition with free nerve endings namely Ruffini end organs and Pacinian corpuscles. ${ }^{[18]}$ The concentration of these mechanoreceptors are greater in number near the ends of ACL attachments and constitutes about $2.5 \%$ of the ligament. ${ }^{[5]}$

\section{Alpha -Smooth Muscle Actin}

In ACL along with the other cells there are presence of alpha actin smooth muscle distributed between the collagen bundles. Murray et al described the presence of smooth muscle cells in the substance of ACL by staining with specific antibody. They suggested that it might be a myofibroblasts because of the morphological and structural 


\section{Banu et al; Htistalagical Structure of Anteriar Cruciate Ligament}

characteristics similar to fibroblasts with alpha actin smooth muscle. Smooth muscle around the blood vessel can be differentiated from smooth muscle cell in the substance of ACL due to distinct arrangement and its presence away from the blood vessels. They described that the cells other than spindle shaped fibroblasts exhibit the immunogen for the alpha-smooth muscle actin antibody. ${ }^{[23]}$

\section{Blood vessels}

The blood vessels were numerous in the epiligament. The blood vessels of about $0.01-0.04 \mathrm{~mm}$ in diameter ran a longitudinal course through the fusiform and ovoid regions. At the intersection of ovoid and fusiform zone nests of capillaries were noted and only few vessels were seen on the spheroid zone. Smaller vessels of about $0.01 \mathrm{~mm}$ were seen in the interfasicular region. ${ }^{[9,23]}$

\section{Functions of Each Component \\ Collagen $^{[6,8]}$}

Type I Collagen: Responsible for high tensile strength

Type II Collagen: seen in fibrocartilagenous zone near the attachments zones. Also in the pericellular matrix around the chondrocytes.

Type III Collagen: seen in loose connective tissue surrounds the collagen bundles and also seen in the attachment zones. It reponsible for pliability of ligament and also anchor the blood vessels to the adjacent matrix and basement membrane. This type of collagen also can be found in the healing process of ligament after an injury.

Type IV Collagen: seen in the basement membrane of blood vessels more concentrated over the proximal and distal region compared with the middle region because of less vascularization on the middle region.

Type VI collagen: It is concentrated more on the proximal and distal attachment zones of rather than middle zone because of high strains in the attachment regions. It follows the arrangement of type III collagen. ${ }^{[6,8]}$

\section{Changes observed in the collagen pattern}

Post exercise collagen density and diameter could be changed results in higher failure loads. ${ }^{[20]}$

Ground substance: It acts as a shock absorber for ACL. Along with water, ground substance was responsible for maintenance of viscoelastic properties of ligament.

Other proteins: There other proteins included laminin, entactin, tenascin and fibronectin were seen in ACL. Fibronectins accounted for $2 \mu \mathrm{m} / \mathrm{mg}$ dry tissue. Fibronectins played a role in normal, healing and growing tissue of ACL. ${ }^{[8]}$

\section{Elastic components}

Oxytalan fibres may be helpful in withstanding the modest multidirectional stress. The Elaunin fibres permit the extreme distance changes during motion and the elastic fibres absorb the recurrent maximal stress. ${ }^{[8,9]}$

\section{Fibroblasts}

It is widely present in the ligament between the collagen bundles. It is responsible for production of collagen.

\section{Mechanoreceptors functions in ACL}

Mechanoreceptors function as transducers converting the tension developed in ACL as physical energy to nervous signal. During knee motion the mechanoreceptors were being stimulated by the intraligamentous tension in collagen bundles. Ruffini endings are slowly adapting in nature. Free nerve endings and plexuses constitutes the pain receptor in joint tissues. The number of mechanoreceptors also contribute for the amount of sensation to the knee joint. More number of mechanoreceptors were seen on the ACL proximal and distal attachments sites. ${ }^{[18,26]}$ Following injury of ACL, small remnants of ACL were seen on the proximal and distal attachment region. Therefore, preservation of stump is important for ACL reconstruction. ${ }^{[25]}$

\section{Histopathology of ACL}

Injuries of ACL

Murray et al studied the injuries of ACL and described changes in the disrupted ACL. They observed the collagen fibres were disrupted at the injury site and four different changes were seen; these are inflammatory phase, an epiligamentous repair phase, a proliferative phase, and a remodelling phase. During the inflammatory phase, disrupted synovial covering and intimal hyperplasia of vessels and crimp pattern loss near the injury site were noted. The repair phase showed more vascularized tissue surrounding the injury site. During the proliferative phase, revascularization with capillaries arranged as groups were observed and on the remodelling phase with decrease in cell number and blood vessel density and retraction of ligament. ${ }^{[31]}$

\section{Mucoid degeneration in ACL}

Mucoid degeneration of ACL was a rare finding in ACL, characterized by the presence of mucoid filled collections in and around the ACL attachments. Histopathological observations suggested of mucoid filled material in the substance of ACL. Gross appearance of mucoid degeneration of ACL looked similar to hypertrophied and bulbous structure with absence of synovial covering. The microscopic appearance showed disrupted collagen fibres admixed with faint purple-coloured mucoid material along with some fine capillaries at places and also mucoid degeneration in loose connective tissue surrounding ACL with Haematoxylin and eosin stain. ${ }^{[15,21,24]}$ ACL mucoid degeneration may be part of general degenerative process of the knee and could be a precursor for osteoarthritis (OA). ${ }^{[27,30]}$

\section{Conclusion}

Overall the arrangement of structures in ACL varied from proximal to distal attachment. The proximal attachment showed ovoid shaped to round cells in between the bundles of collagen fibres. Middle zone showed high density of collagen in between the elastic and oxytalan fibers. There was a special zone of cartilage and fibrocartilage is seen in the substance of ACL along the with the collagen fibers over the impingement region of ACL. Distal zone was solid and consists of low density of collagen with interspersed two types of cells, chondroblasts and ovoid fibroblasts. 
Mechanoreceptors on the ACL maintain the neuromuscular stability of knee joint. Therefore, the mechanorecptors on the remnant injured ACL may be useful for ACL reconstruction. This review may be useful for the orthopaedician and physiotherapist in treating the cases of ACL injuries.

\section{References}

1. Frank CB. Ligament structure, physiology and function. J Musculoskel Neuron Interact 2004;4:199-201.

2. Marieswaran M, Jain I, Garg B, Sharma V, Kalyanasundaram D. A review on biomechanics of anterior cruciate ligament and materials for reconstruction. Appl Bionics Biomech. 2018;2018:1-14

3. Petersen W, Zantop T. Anatomy of the anterior cruciate ligament with regard to its two bundles. Clin Orthop. 2007;454:35-47.

4. Amis AA, Jakob RP. Anterior cruciate ligament graft positioning, tensioning and twisting. Knee Surg Sports Traumatol Arthrosc. 1998;6:2-12.

5. Dhillon MS, Bali K, Prabhakar S. Differences among mechanoreceptors in healthy and injured anterior cruciate ligaments and their clinical importance. Muscles Ligaments Tendons J. 2012;2:38-43.

6. Strocchi R, De Pasquale V, Gubellini P, Facchini A, Marcacci $\mathrm{M}$, Buda R, et al. The human anterior cruciate ligament: histological and ultrastructural observations. J. Anat. 1992;180:515-19.

7. Furumatsu T, Kodama Y, Maehara A, Miyazawa S, Fujii M, Tanaka T, et al. The anterior cruciate ligament-lateral meniscus complex: a histological study. Connective tissue research. 2016;57:91-8.

8. Duthon VB, Barea C, Abrassart S, Fasel JH, Fritschy D, Ménétrey J. Anatomy of the anterior cruciate ligament. Knee Surg Sports Traumatol Arthrosc. 2006;14:204-13.

9. Neurath MF, Stofft E. Characterization of collagenous and elastic fiber systems and microvasculature of human cruciate ligaments: an electronmicroscopic and stereologic study. Clinical Anatomy. 1993;6:146-53.

10. Yelicharla AK, Gajbe U, Singh B. Morphometric study on cruciate ligaments of knee with gender differences: a cadaveric study. Asian Pac. J. Health Sci. 2014;1(3):285-91.

11. Ferretti M, Levicoff EA, Macpherson TA, Moreland MS, Cohen M, Fu FH. The fetal anterior cruciate ligament: an anatomic and histologic study. Arthroscopy. 2007;23:278-83.

12. Triantafyllidi E, Paschos NK, Goussia A, Barkoula NM, Exarchos DA, Matikas TE, et al. The shape and the thickness of the anterior cruciate ligament along its length in relation to the posterior cruciate ligament: a cadaveric study. Arthroscopy. 2013;29:1963-73.

13. Bicer EK, Lustig S, Servien E, Selmi TA, Neyret P. Current knowledge in the anatomy of the human anterior cruciate ligament. Knee Surg Sports Traumatol Arthrosc. 2010;18:107584.

14. Oka S, Schuhmacher P, Brehmer A, Traut U, Kirsch J, Siebold R. Histological analysis of the tibial anterior cruciate ligament insertion. Knee Surg Sports Traumatol Arthrosc. 2016;24:74753.

Copyright: (c) the author(s), publisher. Academia Anatomica International is an Official Publication of "Society for Health Care \& Research Development". It is an open-access article distributed under the terms of the Creative Commons Attribution Non-Commercial License, which permits unrestricted non-commercial use, distribution, and reproduction in any medium, provided the original work is properly cited.

How to cite this article: Banu J, Sontakke Y, Tamgire D, Suma HY. Histological Structure of Anterior Cruciate Ligament - Review. Acad. Anat. Int. 2019;5(1):16-20.

DOI: dx.doi.org/10.21276/aanat.2019.5.1.5
15. Woo SL, Newton PO, MacKenna DA, Lyon RM. A comparative evaluation of the mechanical properties of the rabbit medial collateral and anterior cruciate ligaments. Journal of biomechanics. 1992;25:377-86.

16. Jackson DW, Arnoczky SP. The anterior cruciate ligament: current and future concepts. Lippincott Williams \& Wilkins; 1993.

17. Iwahashi T, Shino K, Nakata K, Otsubo H, Suzuki T, Amano $\mathrm{H}$, et al. Direct anterior cruciate ligament insertion to the femur assessed by histology and 3-dimensional volume-rendered computed tomography. Arthroscopy. 2010;26:13-20.

18. Zimny ML, Schutte M, Dabezies E. Mechanoreceptors in the human anterior cruciate ligament. The Anatomical Record. 1986;214:204-9

19. Wang JH, Lee BH. Mediolateral differences of proteoglycans distribution at the ACL tibial footprint: experimental study of 16 cadaveric knees. BioMed research international. 2018;2018:1-6.

20. Cabaud HE, Chatty A, Gildengorin V, Feltman RJ. Exercise effects on the strength of the rat anterior cruciate ligament. Am J Sports Med. 1980;8:79-86.

21. Pandey V, Suman CP, Sharma S, Rao SP, Acharya KK, Sambaji C. Mucoid degeneration of the anterior cruciate ligament: management and outcome. Indian $\mathrm{J}$ Orthop. 2014;48:197.

22. Yahia LH, Drouin G. Collagen structure in human anterior cruciate ligament and patellar tendon. Journal of materials science. 1988;23:3750-5.

23. Murray MM, Spector M. Fibroblast distribution in the anteromedial bundle of the human anterior cruciate ligament: The presence of $\alpha$-smooth muscle actin-positive cells. Journal of orthopaedic research. 1999;17:18-27.

24. Clark JM, Sidles JA. The interrelation of fiber bundles in the anterior cruciate ligament. Journal of orthopaedic research. 1990;8:180-8.

25. Adachi N, Ochi M, Uchio Y, Iwasa J, Ryoke K, Kuriwaka M. Mechanoreceptors in the anterior cruciate ligament contribute to the joint position sense. Acta Orthopaedica Scandinavica. 2002;73:330-4.

26. Schultz R, Miller D, Kerr C, Micheli L. Mechanoreceptors in human cruciate ligaments: a histological study. Journal of bone and joint surgery. 1984;66:1072-6.

27. Hasegawa A, Otsuki S, Pauli C, Miyaki S, Patil S, Steklov N, et al. Anterior cruciate ligament changes in the human knee joint in aging and osteoarthritis. Arthritis \& Rheumatism. 2012 Mar;64(3):696-704.

28. Suzuki D, Otsubo $H$, Watanabe $T$, Kamiya $T$, Nagoya $S$, Yamashita T, et al. Ultrastructure of the three anterior cruciate ligament bundles. Clinical Anatomy. 2015;28:910-6.

29. Skelley NW, Castile RM, York TE, Gruev V, Lake SP, Brophy RH. Differences in the microstructural properties of the anteromedial and posterolateral bundles of the anterior cruciate ligament. Am J Sports Med. 2015;43:928-36.

30. Cushner FD, La Rosa DF, Vigorita VJ, Scuderi GR, Scott WN, Insall JN. A quantitative histologic comparison: ACL degeneration in the osteoarthritic kneel. The Journal of arthroplasty. 2003;18:687-92.

31. Murray MM, Martin SD, Martin TL, Spector M. Histological changes in the human anterior cruciate ligament after rupture. JBJS. 2000;82:1387-97. 